\title{
Cluster approach to formation of nitrogen-rare gas cryoalloys
}

\author{
A.A. Solodovnik, V.V. Danchuk, and N.S. Mysko \\ B. Verkin Institute for Low Temperature Physics and Engineering of the National Academy of Sciences of Ukraine \\ 47 Lenin Ave., Kharkov 61103, Ukraine \\ E-mail: solodovnik@ilt.kharkov.ua
}

Received December 28, 2012

\begin{abstract}
Structure of solid binary $\mathrm{N}_{2}-\mathrm{Kr}$ and $\mathrm{N}_{2}-\mathrm{Ar}$ solutions were studied by transmission electron diffraction (THEED). The samples were prepared in situ by deposition of gaseous mixture onto an Al substrate cooled to $20 \mathrm{~K}$. The lattice parameters for low concentrations of one of the components were measured. The relative change of the lattice parameter per unit concentration is determined for $\mathrm{N}_{2}$ impurity in $\mathrm{Kr}$ and $\mathrm{Ar}$ as well as for $\mathrm{Kr}$ and $\mathrm{Ar}$ in $\mathrm{N}_{2}$. Analysis of the concentration dependence of the lattice parameters for low fractions of both components was performed within a cluster model in the three-particle approximation.
\end{abstract}

PACS: $78.55 . \mathrm{Kz}$ Solid organic materials;

61.05.J- Electron diffraction and scattering.

Keywords: electron diffraction, cryocrystalls, cluster approach, alloys.

\section{Introduction}

Solutions of cryocrystalls consisting of linear molecules with atomic components are of considerable interest as convenient model systems for solving many problems including the disordered solids, orientational glass states, phase transitions [1,2]. Experimental information on the structure and the concentration dependence of the lattice parameters of solutions can be useful for a critical check of the existing theories and a creation of new descriptions. In some cases Vegard's law and Prigogine theory, which take into account the additional variation of volume, associated with the mixing energy, are insufficient. In this connection, new approaches are needed. One of the deviations from ideal solution theory manifests itself through the anomalous behavior of the lattice parameter as a function of composition. In a review article [3] the available data on diluted binary alloys of rare gases (in particular $\mathrm{Ar}$ and $\mathrm{Kr}$ ) with diatomics $\left(\mathrm{N}_{2}, \mathrm{CO}\right)$ have been systemized and analyzed. For low fractions, when most of the impurity particles are singles, the authors suggested a theory which allowed calculation of the excess volume per impurity and compared their conclusions with experimental data. The concept of a set of smaller clysters (singles, pairs, and triples) has been applied [4] to the problem of the thermodynamics and kinetics of ortho-para mixtures of solid hydrogen. A similar approach was used [5] in an analysis of the thermal properties of $J=1$ with $J=0$ solid mixtures of the hydrogen isotopes. The idea of solitary impurities have been successfully applied in thermodynamic effects of molecular impurities $\left(\mathrm{N}_{2}, \mathrm{CO}, \mathrm{O}_{2}\right)$ in rare gas $(\mathrm{Ar}, \mathrm{Kr})$ matrices for strong (1 mol\%) dilutions [6,7].

The cluster approach has been further developed [8,9] within a semiquantitative theory in order to explain the unusual concentration dependence of the lattice parameter in $\mathrm{CO}_{2}-\mathrm{Ar}$ alloys. This theory takes into account not only the isotropic interaction between particles, but also the presence of anisotropic forces in the molecular matrices. The curve was calculated within a model of pair and triple clusters of Ar impurity. In a recent study on the $\mathrm{CO}_{2}-\mathrm{Kr}$ solutions [10] unusual nonmonotonic dependence of the lattice parameter on composition was analyzed in a new approach. Theoretical analysis considering three mechanisms of interaction of $\mathrm{Kr}$ cluster with crystalline environment of $\mathrm{CO}_{2}$ matrix was given for $\mathrm{CO}_{2}$ cryosolid doped $\mathrm{Kr}$. In this manner the experiment was in a good agreement with the theory.

The aim of this research was to apply the available cluster approach [10] in a semiquantitative analysis of the lattice parameter as a function of the respective impurity fraction. The choice of the $\mathrm{N}_{2}-\mathrm{Ar},-\mathrm{Kr}$ systems was dictated by the following considerations. The use of diatomic molecules as the molecular component has some peculiarities. First, the specific feature of nitrogen-rare gas systems, as compared to $\mathrm{CO}_{2}$-inert gas alloys is the presence of the single-phase region at the both sides of the phase diagram. In the area of 
homogeneous solutions either fcc or $\mathrm{Pa} 3$ structure prevails depending on composition. Therefore there is a possibility to study the different kind of matrix. Second, the anisotropic interaction between particles in these alloys is noticeably weaker than these in the $\mathrm{CO}_{2}$ based solutions, and so the dissolution of spherically symmetric inert gas atoms in the $\mathrm{N}_{2}$ matrices not so extremely influence on concentration dependence of lattice parameter as it was in $\mathrm{CO}_{2}$ alloys. The structure of the binary alloys of nitrogen with inert gases has been studied earlier [11-15], however there are discrepancies between experimental data and calculations.

\section{Experimental procedure}

The structural characteristic of binary $\mathrm{N}_{2}$-inert gas alloys was studied by transmission electron diffraction (THEED) on a standard instrument equipped with a helium cryostat. The method has been described earlier in sufficient details [10]. We will mention only the details relevant to the present work. The samples were grown in situ by depositing gaseous mixtures from a vessel at room temperature on $\mathrm{Al}$ substrates at $20 \mathrm{~K}$. The deposition regime was chosen in order to obtain random distributions of impurity. The Al film served also as the internal standard. The error in the lattice parameter measurements was within $0.1 \%$. The concentration in the gas phase was determined by measuring the partial pressure of components with SETRA differential pressure transducer. The total amount of impurity in gaseous components was below $0.01 \%$. The rate of deposition of the mixture was $4-5 \AA /$ s. Observations were carried out in the temperature range from $5 \mathrm{~K}$ to the sublimation point and the recordings at $T=20 \mathrm{~K}$.

The alloy samples were polycrystalline films that did not show any marked signs of a texture. The presence of a single set of reflections, the low background and no extra broadening of the diffraction lines proved good homogeneity of the solutions. The diffraction patterns for $\alpha-\mathrm{N}_{2}$ based alloys corresponded to the Pa3 symmetry, characteristic of the low-temperature phase of nitrogen. In the specimens with low concentrations of $\alpha-\mathrm{N}_{2}$ the solid solutions had fcc structure. Subsequent cooling of the samples from $20 \mathrm{~K}$ to liquid helium temperature and heating up to sublimation point did not lead to any changes in their crystallographic structure.

\section{Experimental results}

Before analyzing the experimental results we present some theoretical considerations suggested earlier [10]. There are the conditions of applicability of this approach: Distribution of impurities is close to random. The number of impurity singles and pairs are estimated exactly while the number of the lager clusters is taken into account in the followings effective way [10]. Within this effective threeparticle approximation the relative number of clusters as function of the molar concentration $x$ of the impurity is as shown in Fig. 1, and calculated by expressions (1)-(3):

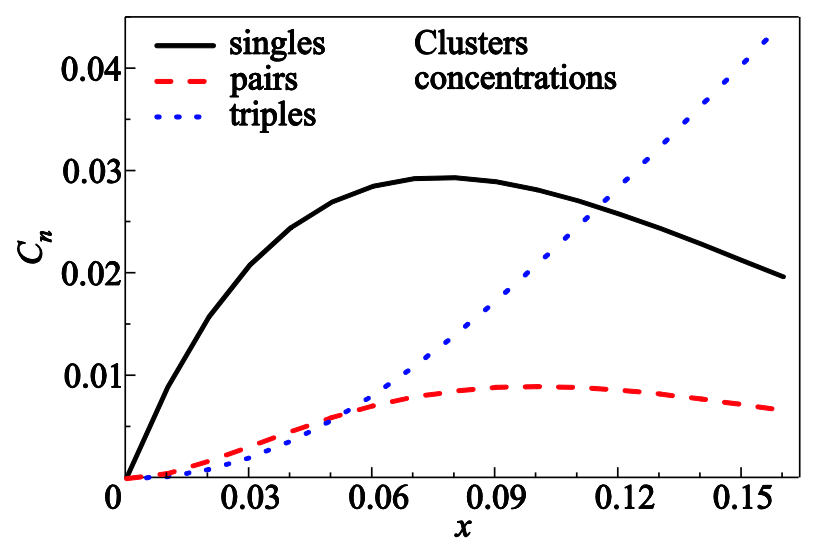

Fig. 1. Relative number of clusters versus the total concentrations of the substitution impurity $C_{n r}$ in the three-particle approximations.

$$
\begin{gathered}
C_{1 r}=x(1-x)^{12}, \\
C_{2 r}=6 x^{2}(1-x)^{18}, \\
C_{3 r}=\frac{x-\left(C_{1 r}+2 C_{2 r}\right)}{3},
\end{gathered}
$$

where $C_{1 r}, C_{2 r}, C_{3 r}$ are the concentrations of singles, pairs and triples clusters, respectively [10].

The relative variation of the lattice parameter per unit concentration can be:

$$
\Delta=\frac{a(x)-a(0)}{a(0) x},
$$

where $x$ is the as low as possible concentration of impurities, $a(x)$ is lattice parameter of the solution for concentration $x$. Actually, $\Delta$ can be treated as the reaction of the lattice on the introduction of the isolated singles of the impurity. From obtained experimental data for compositions of $5 \mathrm{~mol} \%$ impurity the estimates gave such values $\Delta=0.02$ for $\mathrm{Kr}$ in $\mathrm{N}_{2} ; 0.06$ for $\mathrm{N}_{2}$ in $\mathrm{Kr} ;-0.02$ for $\mathrm{Ar}$ in $\mathrm{N}_{2}$; and 0.04 for $\mathrm{N}_{2}$ in Ar.

Since the structure of alloys is of cubic symmetry the number of nearest neighbors $N_{n}$ in a cluster of $n$ particles is $N_{n}=12,18,22$, respectively for $n=1,2,3$. The two-dimension visualization of different clusters nearest neighbors is presented on Fig. 2. The relative alteration of the lattice parameter $\Delta_{n}$ per particle of a cluster of type $n$ is $\Delta$, $3 \Delta / 4$ and $11 \Delta / 18$ for $n=1,2,3$, respectively.

For subsequent calculations of the variation of lattice parameter we used the expression

$$
\frac{a(x)-a(0)}{a(0)}=\sum_{n=1,2,3} \Delta_{n} C_{n r}(x),
$$

where $C_{n r}$ are the concentrations different clusters. Obtained values for only singles $(n=1)$, singles with pairs ( $n=1$ and $n=2$ ), and all three types of clusters are pre- 


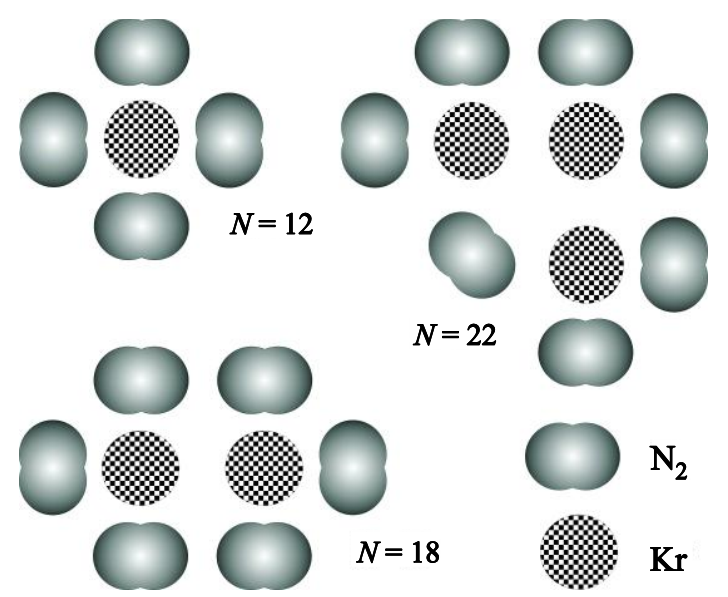

Fig. 2. The nearest neighbors two-dimension visualizations for different types of clusters.

sented in Fig. 3. One can see that account of pairs and triples influences substantially the lattice parameter values less than especially for concentrations $x>5 \mathrm{~mol} \%$.

The sign of the changes of the lattice parameter depends on whether the interaction between impurities and regular particles is attractive or repulsive. Making use of the pairwise Lennard-Jones potential quantities $\sigma$ and $\varepsilon$ [1] for $\mathrm{Kr}$, Ar, and $\mathrm{N}_{2}$ as well as the experimental nearest neighbor distances, we evaluated the relevant interaction parameter as shown in Table 1.

The interaction between an atomic impurity and a molecular matrix comprises two contributions, the central and anisotropic ones. The former can lead to shrinking or expanding effects, depending on the parameters involved, whereas the anisotropic interaction always makes the lattice of "atom-in-molecular-matrix" alloys expand, since the corresponding part of the attractive anisotropic interaction disappears.

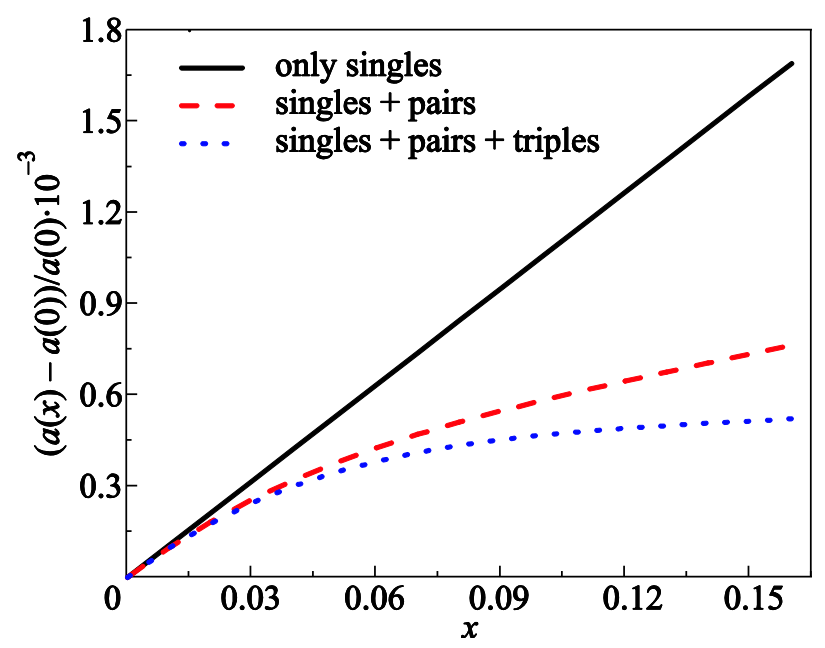

Fig. 3. The theoretical dependence of the lattice parameter alteration versus the impurity concentration.
Table 1 . The type of interaction between impurities and matrices

\begin{tabular}{c|c|c|c}
\hline \hline Alloys & $R_{\min } \AA$ & $R_{\text {interpart }} \AA$ & Result \\
\hline \hline Ar in $\mathrm{N}_{2}$ & 3.977 & 4.03 & attraction \\
$\mathrm{Kr}$ in $\mathrm{N}_{2}$ & 4.1 & 4.06 & repulsion \\
$\mathrm{N}_{2}$ in $\mathrm{Ar}$ & 3.977 & 3.83 & repulsion \\
$\mathrm{N}_{2}$ in $\mathrm{Kr}$ & 4.1 & 4.05 & repulsion \\
\hline \hline
\end{tabular}

The theoretical dependences of lattice parameter versus concentration are calculated by the expression:

$$
a(x)_{\text {theor }}=a(0)+\Delta C_{1 r}+\Delta \frac{3 C_{2 r}}{8}+\Delta \frac{11\left(x-C_{1 r}-C_{2 r}\right)}{54}
$$

and presented in Figs. 4(a) and 4(b) by triangles for $\mathrm{N}_{2}-\mathrm{Kr}$ and $\mathrm{N}_{2}-\mathrm{Ar}$ systems, respectively, for both sides of the phase diagrams.

The experimental data of the concentration dependencies of lattice parameters were measured at $20 \mathrm{~K}$ and the results are presented in Fig. 4 as circles and squares, the dashed line corresponds to the dependence for ideal solution.

\section{$\alpha-\mathbf{N}_{2}-\mathbf{K r}$}

As can be seen in Fig. 4(a), the values of the parameter obtained experimentally lie higher, than Vegard's line and changes smoothly as a function of composition. Despite

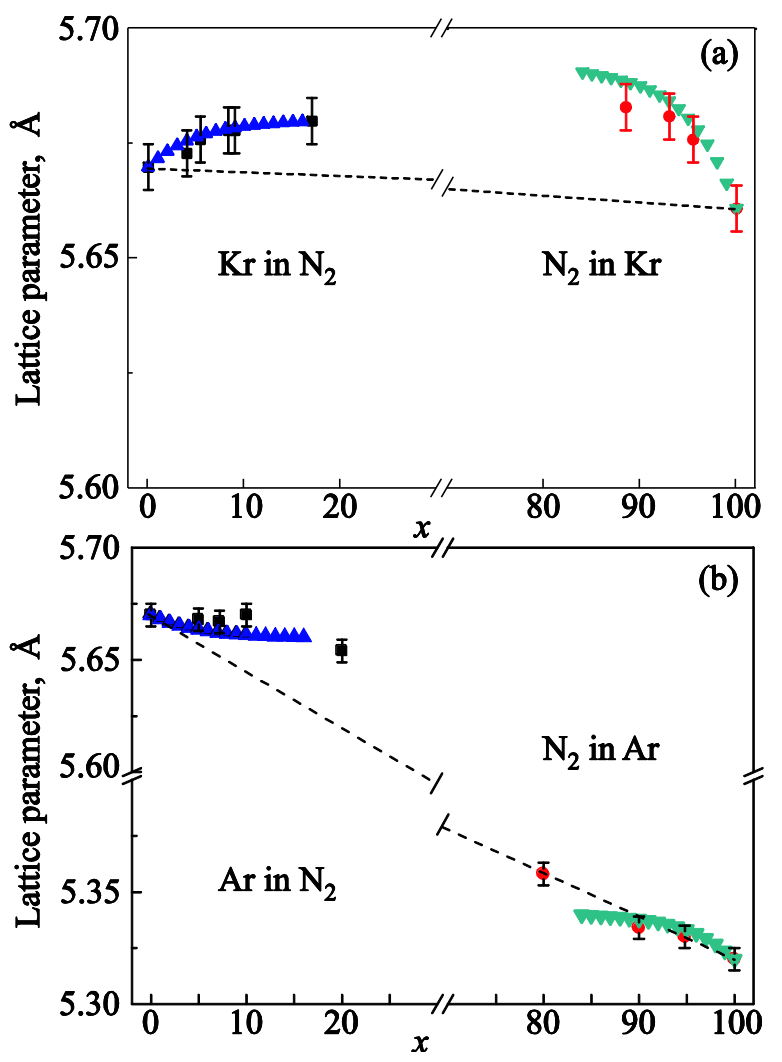

Fig. 4. Concentration dependence of the lattice parameter $\mathrm{N}_{2}-\mathrm{Kr}$ (a) and $\mathrm{N}_{2}-\mathrm{Ar}$ (b). $T=20 \mathrm{~K}$. The circles and squares are experimental data. The triangles are theoretical calculations. 
the closeness of interparticle distances in lattice of pure components the experimental points substantially deviate from Vegard's line and on the whole the picture is asymmetric.

On the side of nitrogen as the krypton content increases to $7 \%$ the lattice parameter growth sharply, since in this range it is the singles which dominate and affect most efficiently the matrix, while the central and anisotropic contribution have the same sign. Further increase of the Kr content slows down strongly the concentration related expansion of the lattice. This experimental finding is in line with the inference of our cluster approach that effect (per particle involved) of larger clusters is appreciably weaker than that of singles. Here we once again note that for the case of $\mathrm{Kr}$ in $\mathrm{N}_{2}$ the contributions of the central and anisotropic parts have the same sign. That means that their net contribution to the variation of the average lattice parameter is correctly accounted for using the quantity $\Delta$ and proves that the cluster approach describes well the experimental observations.

In the case of the molecular impurity in an atomic matrix the fact of the small difference in the lattice parameter is not evidently. The molecules of nitrogen have its changed anisotropic interaction and it leads to the increase of lattice parameter in concentration interval 0-7 mol\% for Kr-based solutions. In atomic matrix (concentration range 7-12 mol\% $\mathrm{N}_{2}$ ) the pair and triple $\mathrm{N}_{2}$ clusters is subjected to compression. It follows from these facts. The compressibility of pure solid components at $T=20 \mathrm{~K}$ is $\chi=2.88 \cdot 10^{-11} \mathrm{~cm} /$ dyne for krypton [16] and $\chi=4.64 \cdot 10^{-11} \mathrm{~cm} /$ dyne for nitrogen [1]. In solid nitrogen the bonds are weaker than in $\mathrm{Kr}$. The compression results in an additional negative contribution to the expansion of the lattice due to the presence of large $\mathrm{N}_{2}$ clusters. Furthermore the cluster formation initiates the noncentral interaction between $\mathrm{N}_{2}$ molecules that also leads to negative contribution. These two factors are behind the fact that within the fraction range $7-15 \mathrm{~mol} \%$ the calculated dependence goes above experimental points.

\section{$\alpha-\mathrm{N}_{2}-\mathrm{Ar}$}

The concentration dependence of the lattice constant for $\alpha-\mathrm{N}_{2}-\mathrm{Ar}$ system at $T=20 \mathrm{~K}$ is given in Fig. 4(b). Should be note, the difference in lattice parameter of $\alpha-\mathrm{N}_{2}$ and $\mathrm{Ar}$ is $7 \%$. On the side of nitrogen the lattice parameter of solution as a function of argon concentration behaves as a weak dependence with increasing content of $\mathrm{Ar}$ in the range $0-10 \mathrm{~mol} \% \mathrm{Ar}$. The experimental values of the parameter lie higher than the Vegard's dashed line and even have a different sign as compared of ideal solutions. For the solution in which nitrogen predominates the two opposing processes are operative. On the one hand, the introduction of smaller argon atoms must lead to a compression of the crystal. The negative contribution to the expansion of the lattice is due to the attractive interaction between particles. The interparticle distance $4.03 \AA$ is more than the distance $R=3,977 \AA$ of the minimum of the interaction energy as estimated from the values of the Lennard-Jones parameters for $\alpha-\mathrm{N}_{2}$ and Ar. On the other hand, the vanishing of the anisotropic forces due to the substitutional atomic impurities causes the expansion of the lattice. At argon concentrations above $10 \mathrm{~mol} \%$ the attractive interaction becomes more influential in the result of the competition. The vanishing anisotropic interaction becomes less powerful factor, and the noticeable decrease of the solution parameter is observed. A peculiar fact of this system is that the central and anisotropic interaction components have different signs and, because of the inevitable competition, their efficiencies vary with impurity concentration. Therefore, the assessments based solely on a single parameter $\Delta$ are incorrect over the entire concentration range under discussion.

For Ar-based solutions the concentration dependence of the lattice constant is close to that predicted Vegard's rule. It is difficult to explain how the $\mathrm{N}_{2}$ molecules are located in the Ar matrix. The following deceleration of lattice parameter of $\mathrm{N}_{2}$ in Ar cryoalloys in concentration range from 7 to $15 \mathrm{~mol} \%$ is explained similarly to $\mathrm{N}_{2}$ in $\mathrm{Kr}$ system. The pair and triple clusters of nitrogen are subjected to compression, because the bonds in solid nitrogen are weaker than in Ar.

The coefficients of compressibility at $20 \mathrm{~K}$ are: for nitrogen $\chi=4.64 \cdot 10^{-11} \mathrm{~cm} /$ dyne, for Ar $\chi=3.73 \cdot 10^{-11} \mathrm{~cm} /$ dyne [17]. Furthermore the cluster formation initiates the noncentral interaction between $\mathrm{N}_{2}$ molecules that also leads to decreasing of lattice parameter growth.

\section{Conclusion}

1. Structure characteristics of binary solid $\mathrm{Kr}$ in $\alpha-\mathrm{N}_{2}$, Ar in $\alpha-N_{2} ; N_{2}$ in $\mathrm{Kr}$ and $\mathrm{N}_{2}$ in Ar solutions have been studied for impurity contents below $15 \mathrm{~mol} \%$. The samples were grown and studied at $T=20 \mathrm{~K}$.

2. The respective quantities $\Delta$, which are the relative lattice parameter change per unit impurity fraction. The notions of central and anisotropic contribution to the above mentioned change have been introduced.

3. Within the cluster approach the lattice parameter versus impurity fraction dependence were analyzed for low impurity fractions. It is shown that the simple cluster approach is completely valid only for $\mathrm{Kr}$ in $\mathrm{N}_{2}$. The strongly varying competition between the central and anisotropic contributions for $\mathrm{Ar}$ in $\mathrm{N}_{2}$ leaves doubts concerning exact applicability of the cluster approach based solely on a unique parameter Ar over the entire concentration range studied.

4. In order to use the cluster approach adequately, further improvements are needed, including account of the interaction between impurities within larger clusters.

The authors are grateful to M.A. Strzhemechny for discussions of the problem. 
1. The Physics of Cryocrystals, V.G. Manzhelii, Yu.A. Freiman, M.L. Klein, and A.A. Maradudin (eds.), AIP Press, Woodbury (1997).

2. V.G. Manzhelii, A.I. Prokhvatilov, I.Ya. Minchina, and L.D. Yantsevich, Handbook of Binary Solutions of Cryocrystals, Begell House, New York (1996).

3. M.A. Strzhemechny, A.I. Prokhvatilov, and L.D. Yantsevich, Physica B 198, 267 (1994).

4. J.F. Jarvis, H. Meyer, and D. Ramm, Phys. Rev. 178, 1461 (1969).

5. I.Ya. Minchina, M.I. Bagatskii, V.G. Manzhelii, and A.I. Krivchikov, Fiz. Nizk. Temp. 10, 1051 (1984) [Sov. J. Low Temp. Phys. 10, 549 (1984)].

6. A.I. Krivchikov, M.I. Bagatskii, V.G. Manzhelii, I.Ya. Minchina, and P.I. Muromtsev, Fiz. Nizk. Temp. 14, 1208 (1988) [Sov. J. Low Temp. Phys. 14, 667 (1988)].

7. P.I. Muromtsev, M.I. Bagatskii, V.G. Manzhelii, I.Ya. Minchina, and A.I. Krivchikov, Fiz. Nizk. Temp. 16, 1058 (1990) [Sov. J. Low Temp. Phys. 16, 616 (1990)].

8. M.A. Strzhemechny, S.I. Kovalenko, and A.A. Solodovnik, Fiz. Nizk. Temp. 18, 1387 (1992) [Low Temp. Phys. 18, 967 (1992)].
9. M.A. Strzhemechny, A.A. Solodovnik, and S.I. Kovalenko, Fiz. Nizk. Temp. 24, 889 (1998) [Low Temp. Phys. 24, 669 (1998)].

10. V.V. Danchuk, A.A. Solodovnik, and M.A. Strzhemechny, Fiz. Nizk. Temp. 36, 321 (2010) [Low Temp. Phys. 36, 254 (2010)].

11. C.S. Barrett and L. Meyer, J. Chem. Phys. 42, 107 (1965).

12. S.I. Kovalenko, I.N. Krupskii, and A.A. Solodovnik, Fiz. Nizk. Temp. 2, 781 (1976) [Sov. J. Low Temp. Phys. 2, 385 (1976)].

13. S.I. Kovalenko, I.N. Krupskii, and A.A. Solodovnik, Fiz. Nizk. Temp. 7, 1445 (1981) [Sov. J. Low Temp. Phys. 7, 700 (1981)].

14. A.I. Prokhvatilov and L.D. Yantsevich, Fiz. Nizk. Temp. 10, 517 (1984) [Sov. J. Low Temp. Phys. 10, 270 (1984)].

15. L.D. Yantsevich, A.I. Prokhvatilov, I.N. Krupskii, and A.S. Barylnik, Fiz. Nizk. Temp. 12, 300 (1986) [Sov. J. Low Temp. Phys. 12, 170 (1986)].

16. D.L. Losee and R.O. Simmons, Phys. Rev. 172, 944 (1968).

17. V.G. Gavrilko, PhD Thesises, FTINT, Kharkov (1969). 